\title{
DIFFUSION OF ALKALI SPECIES IN POROUS TUNGSTEN SUBSTRATES USED IN CONTACT-IONIZATION SOURCES*
}

\author{
E. Chacon-Golcher ${ }^{1}$, J.W. Kwan ${ }^{2}$ and E. C. Morse ${ }^{3}$ \\ ${ }^{1}$ LANL, ${ }^{2}$ LBNL, ${ }^{3}$ UC-Berkeley
}

\begin{abstract}
Contact ionization (doped) sources used in current Heavy Ion Fusion (HIF) experiments consist of a porous tungsten substrate doped with an alkali carbonate. During the early stages of the heating cycle $\left(\mathrm{T} \sim 600{ }^{\circ} \mathrm{C}\right)$, the carbonate breaks down and releases the alkali atoms that then diffuse through the substrate. At the emitter surface there is a balance between the fast desorption rate of the alkali atoms (mostly as neutrals) and the slower replenishment rate from the substrate by diffusion. Timeresolved measurements of neutral particle evaporation rates at the emitter surface have been used to estimate the effective diffusion coefficient (D) that characterizes the migration of alkali species in the substrate. These estimates are consistent with the observed source lifetimes (tens of hrs.) and establish the alkali migration in the bulk as a diffusion-limited process. The measurements suggest that the faster migration rates $\left(\mathrm{D} \approx 10^{-5}-10^{-6} \mathrm{~cm}^{2} / \mathrm{s}\right)$ occur early during the heating cycle when the dominant species are the neutral alkali atoms. At operating temperatures there is a slower migration rate $\left(\mathrm{D} \approx 10^{-7} \mathrm{~cm}^{2} / \mathrm{s}\right)$ due to the dominance of ions, which diffuse by a slower surface diffusion process.
\end{abstract}

\section{INTRODUCTION}

Original studies carried out in the field of surface physics of alkali atoms on tungsten can be traced back to the definitive work by Langmuir [1]. Subsequent technological applications of those fundamental principles include space propulsion applications and their continued use as a source of heavy ions for inertial fusion energy drivers. Despite the wealth of information accumulated in the ion thruster research, there have been diverse needs to explore the performance of these ion sources for HIF research given the very different regimes of operation present in these two applications. Among the differences for HIF we have: higher ion current density demand, desire for lower neutral particle emission, and a requirement of a lower effective ion temperature (low beam emittance). Previous investigations carried out in LBNL have shown the capability of these ion sources to deliver $\mu$ s-length pulses of approximately $100 \mathrm{~mA} / \mathrm{cm}^{2}$ for a potassium beam under typical experimental conditions. This and a description of the basic preparation of these ion sources are elaborated in a previous report [2]. One of the main research interests in HIF applications relate to the ion source lifetime and the atomic processes that

\footnotetext{
*Work supported by the Office of Fusion Energy, US DOE under contract No. DE-AC03-76SF00098.
}

determine it. This report summarizes a simple methodology applied to understand the importance of diffusive processes in the performance of this type of sources. Using previously measured [3] rates of neutral alkali atom desorption to match theoretical curves it was possible to estimate the diffusion constant of the alkali species in the porous tungsten substrate, in good agreement with the observed ion source lifetimes.

\section{ONE DIMENSIONAL DIFFUSION MODEL}

\subsection{Physics Issues}

It is known that the mean residence lifetime $(\tau)$ of an alkali atom on a tungsten surface decreases exponentially with temperature (T). For instance, in the Ahrrenius-type relation

$$
\tau=\tau_{0} e^{Q / k T}
$$

the parameter $\tau 0$ has a magnitude of approximately 10-13 $\mathrm{s}$ and the energy desorption parameter, $\mathrm{Q}$, is on the order of $1 \mathrm{eV}$. These values amount to desorption times of a fraction of a nanosecond over the range of temperatures of interest for ion emission. The porous tungsten substrates used in HIF experiments are doped with an initial amount of alkali atoms (Cs or $\mathrm{K}$ ). Considering the desorption times, it can be concluded that it is the slow diffusion of the alkalis towards the emitting surface what determines the observed ion source lifetimes.

Even when considering the case of the diffusion of a single species, the process is highly dynamical and somewhat complicated. Initially, the concentration of potassium or cesium in the emitter substrate is relatively large, given the dose of carbonate used, which provides enough alkali atoms to have a full monolayer of coverage throughout the internal surfaces of the emitter. It is expected that the initial distribution of the alkali atoms will become more uniform upon the breaking down of the chemical compounds and the natural diffusion of the alkalis in the bulk. Several stages can be identified:

I. An initial stage of an alkali rich bulk. This stage should be identified at temperatures beyond $600{ }^{\circ} \mathrm{C}$. At this stage, the high concentration of alkali atoms highly favors their existence as neutral particles which have a smaller surface affinity with the tungsten than the alkali ions. Therefore, a neutral particle diffusion dominated process is expected. This does not occur at very fast rates 
since this stage usually happens while the emitter is at relatively low temperatures during the warm up cycle.

II. A mid-stage where sufficient alkali atoms have been desorbed so that there starts to appear a significant ion population. The neutral atoms will continue to diffuse through like in a Knudsen-type flow (given that the mean free path of the atoms is much larger than the characteristic dimensions of the pores), being slowed down by the fact that the gas atoms do not elastically collide with the emitter internal surfaces and bounce right off, but become adsorbed, spend some time on the surface and then become desorbed again. The ions, on the other hand, due to their binding force to the surface, are limited to a slower diffusion speed, as their movement is through surface migration along the grain surfaces.

III. A final stage where the alkali content is sufficiently low to favor the existence of ions, which as said in point b., move by surface diffusion. It is at this stage that the source is fully operational and capable of its higher current yields. It is also this slow surface diffusion mechanism that explains the observed lifetimes of the surface ionization sources.

The above stages may be interpreted from neutral desorption rate data during the heating cycles of doped tungsten substrates [3]. Given the diffusion dynamics described above, the estimated effective diffusion constants ('D') at different temperatures do not scale simply with an Ahrrenius-type formula.

\subsection{Mathematical Model and Data Fitting}

The porous $\mathrm{W}$ substrates used in these measurements were small cylindrical pellets $($ diameter $=0.6 \mathrm{~cm}$, length $=$ $0.64 \mathrm{~cm}$ ) surrounded by a molybdenum holder. Only one of the circular ends was exposed to the vacuum chamber. Once the dopant was included in the pellet, the diffusive flow would only occur in a single direction. The equation that relates the concentration ' $\mathrm{c}$ ' with the distance from the back of the emitter ' $x$ ' and the time ' $t$ ' is the known diffusion equation.

$$
\frac{\partial c(x, t)}{\partial t}-D \frac{\partial^{2} c(x, t)}{\partial x^{2}}=0
$$

The solution of this equation was subject to the following boundary conditions.

$$
\begin{aligned}
& c(x, 0)=c_{0} \\
& \left.\frac{\partial c}{\partial x}\right|_{x=0}=0 \\
& c(L, t)=0
\end{aligned}
$$

which represent the conditions of initial uniform concentration, no flow at the back end of the emitter and an artificial boundary condition of zero concentration at $\mathrm{x}$
$=\mathrm{L}$ respectively. $\mathrm{L}$ is just at a very small distance from the actual emitting surface to which the experimental data was fitted.

The exact solution to this problem is given by equation (6). This can be obtained in direct analogy with a heat diffusion problem [4].

$$
\frac{c}{c_{0}}=\sum_{n=1}^{\infty} C_{n} \exp \left(-\frac{\varsigma_{n}^{2} D t}{L^{2}}\right) \cos \left(\frac{\varsigma_{n} x}{L}\right)
$$

Here ' $\mathrm{c}_{0}$ ' is the concentration at the beginning of the interval in which the data is fit, and the coefficients ' $\mathrm{C}_{\mathrm{n}}$ ' and ' $S_{n}$ ' are related by equations (7) and (8). The condition in equation (8) reflects the fact that the desorption rate of the particles at the surface is much faster than the diffusion rate towards the surface.

$$
\begin{gathered}
C_{n}=\frac{4 \sin \left(\varsigma_{n}\right)}{2 \varsigma_{n}+\sin \left(2 \varsigma_{n}\right)} \\
\varsigma_{n} \tan \left(\varsigma_{n}\right)=\infty
\end{gathered}
$$

Figure 1. shows the curves predicted by equation (6) using the first four terms of the series. The graph shows the decrease in concentration in the bulk with time.

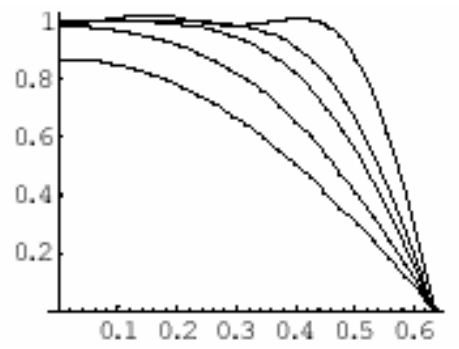

Figure 1: Concentration profile (with respect to original value) as a function of position (in $\mathrm{cm}$ ) with increasing time as a parameter (lower concentration curves).

Equation (6) was used to fit the experimental measurements of neutral atom desorption of cesium and potassium atoms. These data exist for a collection of operating temperatures and at different points of the heating cycle of the emitter [3]. After applying an appropriate normalization factor to adjust the magnitude, the curve is fitted by varying a single parameter, the effective diffusion coefficient ' $\mathrm{D}$ '.

The clear assumption of this method is that the neutral particle desorption rate at the emitter surface varies linearly with the concentration of particles at the surface.

Figure 2 shows an example the fitting of experimental data with the theoretical model. The discontinuities of the data correspond to the background level signal, when the detector was moved out of the line of sight of the emitter. 


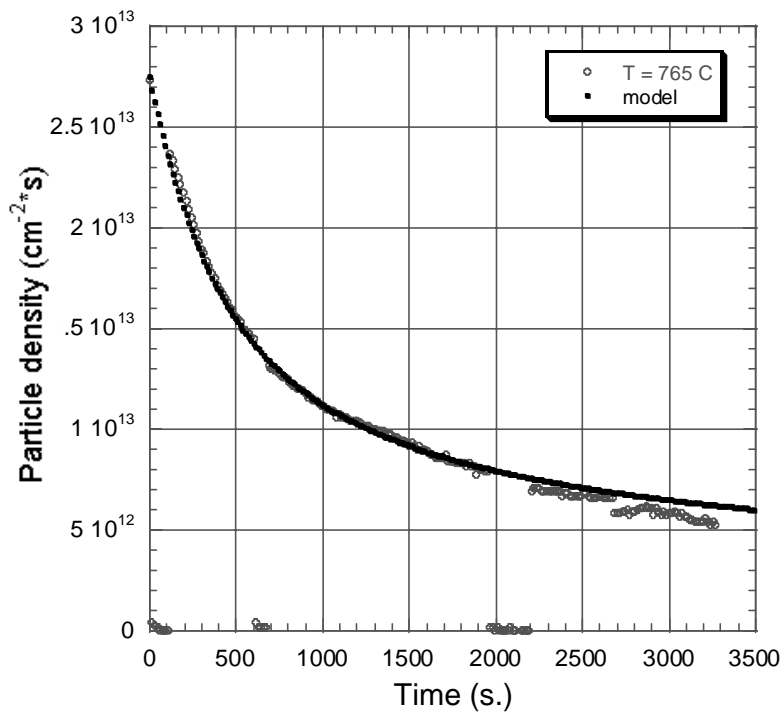

Figure 2. Fitting of the measured neutral particle desorption rate from a cesium source at $\mathrm{T}=765^{\circ} \mathrm{C}$. Effective $\mathrm{D}=1.2 \cdot 10^{-5} \mathrm{~cm}^{2} / \mathrm{s}$.

With a similar procedure a series of effective diffusion coefficients was estimated during the heating cycle of Cs and $\mathrm{K}$ ion sources and at different operating temperatures. A summary is presented in Table 1 . The cells with a gray color represent the temperatures and conditions normally encountered in the operation stage of these ion sources.

\begin{tabular}{ccc}
\hline & \multicolumn{2}{c}{ Diffusion Coefficient $\left(\mathbf{c m}^{2} / \mathbf{s}\right)$} \\
\hline Temperature $\left({ }^{\circ} \mathbf{C}\right)$ & Cesium & Potassium \\
680 & $5.9 \cdot 10^{-06}$ & \\
765 & $1.2 \cdot 10^{-05}$ & \\
800 & $3.7 \cdot 10^{-06}$ & $4.0 \cdot 10^{-06}$ \\
815 & & $5.0 \cdot 10^{-06}$ \\
840 & $1.0 \cdot 10^{-05}$ & \\
885 & $6.0 \cdot 10^{-06}$ & \\
955 & $4.0 \cdot 10^{-06}$ & $4.0 \cdot 10^{-06}$ \\
955 & $1.1 \cdot 10^{-06}$ & $2.0 \cdot 10^{-06}$ \\
955 & $6.5 \cdot 10^{-07}$ & $1.4 \cdot 10^{-06}$ \\
955 & $5.0 \cdot 10^{-07}$ & $3.0 \cdot 10^{-07}$ \\
1,025 & $1.0 \cdot 10^{-06}$ & \\
1,030 & & $1.6 \cdot 10^{-06}$ \\
1,055 & $1.2 \cdot 10^{-06}$ & \\
1,075 & $8.0 \cdot 10^{-07}$ & $1.8 \cdot 10^{-06}$ \\
1,095 & & $1.5 \cdot 10^{-06}$ \\
1,110 & $1.35 \cdot 10^{-06}$ & \\
1,130 & $5.7 \cdot 10^{-06}$ & \\
1,150 & & $1.9 \cdot 10^{-06}$ \\
1,185 & & $3.5 \cdot 10^{-06}$ \\
\hline
\end{tabular}

Table 1. Estimated diffusion constants of Cs and $\mathrm{K}$ through a nominal $12 \mu \mathrm{m}$ grain size porous $\mathrm{W}$ substrate. Several values for $\mathrm{D}$ are listed for the temperature of 955

${ }^{\circ} \mathrm{C}$ as at this temperature the source is allowed to eliminate the surplus of alkali atoms and during a period that lasts $\sim 17$ hours. Diffusion becomes slower with time at this stage. Gray color represents typical operating T's.
In general, the uncertainty of the values can be estimated to \pm 5 in the first decimal place to have curve fits with the type of agreement shown in Figure 2.

\section{DISCUSSION AND CONCLUSIONS}

From the estimates in Table 1 it can be seen how at the lower temperatures, while the diffusion of the particles is dominated by neutral atoms, there is not much sensitivity to ' $\mathrm{T}$ '. This may be explained qualitatively by knowing that the expected faster diffusion rate (at higher $\mathrm{T}$ ) is opposed by the decrease in concentration of alkalis. This decrease results in a larger fraction of particles migrating as ions, which due to their stronger affinity with the surface, migrate at a slower rate. This effect may be more clearly appreciated after the emitters have been allowed to eliminate their surplus alkalis (at $\mathrm{T}=955^{\circ} \mathrm{C}$ ). At this temperature, the diffusion becomes slower with time, until the temperature starts to rise again. Then, there is still not a clear increase of the flow with $\mathrm{T}$ (proportional to ' $\exp (\mathrm{E} / \mathrm{kT})$ ', for some activation energy ' $\mathrm{E}$ ') since the ratio of ions to neutrals starts decreasing with $\mathrm{T}$ as well in accordance with the Saha-Langmuir equation [2].

The estimates of ' $\mathrm{D}$ ' are valid for the specific substrates used in these experiments, i.e. sintered tungsten made with nominal $12 \mu \mathrm{m}$ grain size. While it was found that $\mathrm{D}$ varies with $\mathrm{T}$ and the alkali content of the emitter, the values found are a good indication of the magnitude of $\mathrm{D}$ to be expected in similar conditions.

With regard to ion source lifetimes, consider a Cs source operating at $1,150^{\circ} \mathrm{C}$. Taking a diffusion length of $\mathrm{L}=0.64 \mathrm{~cm}$, the associated time scale would be $\tau_{\mathrm{d}} \sim \mathrm{L}^{2} / \mathrm{D}$ $=(0.64 \mathrm{~cm})^{2} /\left(5.7 \cdot 10^{-6} \mathrm{~cm}^{2} / \mathrm{s}\right) \cong 20 \mathrm{hrs}$. This is consistent with the observed source lifetimes of $\sim 5 \tau_{\mathrm{d}}$. This suggests that for the duty factors used in these experiments, $(\sim 2 \mu \mathrm{s}$, every $20 \mathrm{~s}$, or $\sim 110^{-7}$ ) the main loss mechanism for alkali atoms is indeed neutral particle loss.

With these results is also possible to estimate the replenishment rate at the emitter surface. By assuming a "surface thickness", ' $l$ ', of the order of the grain size, one can propose a formula like (9) for estimating the maximum pulse repetition rate (MPRR) that would allow a particle balance at the surface for a given current density demand sufficient to significantly affect the surface concentration. This is of interest to HIF applications.

$$
\text { MPRR }=\frac{1}{5 \tau_{d}}=\frac{D_{b u l k}}{5 l^{2}}
$$

\section{REFERENCES}

[1] Taylor, J.B. and Langmuir, I. Phys. Rev. 44. No. 6. Sept. 1933. p. 423.

[2] Chacon-Golcher, E. et al. Proceedings of the Particle Accelerator Conference, Chicago, IL. 2001. p. 2102.

[3] Chacon-Golcher, E. et al. Rev. Sci. Instrum. Vol. 73. No. 2. Feb. 2002. p. 1036.

[4] Incropera, F.P. and DeWitt, D.P. Fundamentals of Heat and Mass Transfer. 5th Ed. John Wiley \& Sons. 2002. 